\title{
Evaluación del grado de control del asma en un centro de atención primaria. Un estudio descriptivo
}

\author{
GONZALO ALVEAR T.*, LIDIA FIGUEROA R.**, JAVIERA HURTADO G.** y LUIS MOYANO M.**
}

\section{Assessment of the degree of asthma control in a primary care center}

Achieve a good clinical control by reducing symptoms and management of modifiable risk factors for exacerbations is the main goal of asthma treatment. Our objective was to assess the degree of clinical control and the presence of modifiable risk factors of exacerbations in asthmatic patients controlled in primary care. Data were extracted from clinical records of asthmatics attending their scheduled visit between November 2014 and June 2015. In each visit the Asthma Control Test was performed. 236 visits (183 patients) were analyzed. 10\% had totally controlled asthma, 34\% partially controlled and $56 \%$ uncontrolled. The worst clinical control was significantly associated with greater use of health resources. 92\% had modifiable risk factors of exacerbation, mainly obesity and active smoking.

Key words: Asthma Control. Primary Care.

\section{Resumen}

Lograr un buen control clínico mediante la disminución de los síntomas y el manejo de factores de riesgo modificables de exacerbaciones es el objetivo principal del tratamiento del asma. Nuestro objetivo fue evaluar el grado de control clínico y la presencia de factores de riesgo modificables de exacerbaciones en asmáticos que se controlan en atención primaria. Se extrajeron datos de registros clínicos de los asmáticos que acudieron a su visita programada entre noviembre de 2014 y junio de 2015. En cada visita se realizó el Test de Control del Asma. Se analizaron 236 visitas (183 pacientes). El $10 \%$ tenía el asma totalmente controlada, 34\% parcialmente controlada y $56 \%$ no controlada. El peor control clínico se asoció significativamente con mayor uso de recursos sanitarios. El 92\% tenía factores de riesgo modificable de exacerbaciones, principalmente obesidad y tabaquismo activo.

Palabras clave: Control del Asma. Atención primaria.

\section{Introducción}

El objetivo actual del tratamiento del asma consiste en alcanzar y mantener el control clínico de la enfermedad ${ }^{1}$, el que se compone de dos dominios: a) el control de síntomas y limitaciones del paciente y b) el manejo de los riesgos de mala evolución futura, que incluye las exacerbaciones, el desarrollo de obstrucción fija al flujo de aire y las reacciones adversas a los medicamentos ${ }^{2}$. El nivel de control se refiere al estado actual de la enfermedad, el que puede variar marcadamente en períodos cortos de tiempo y refleja más di- rectamente la efectividad de las intervenciones terapéuticas ${ }^{3}$. Como tanto los pacientes como los médicos tratantes generalmente sobrestiman el grado de control de la enfermedad ${ }^{4}$, se han desarrollado una serie de herramientas simples de aplicar en la práctica diaria para identificar a los pacientes con asma mal controlada. Las más utilizadas son los cuestionarios de GINA, el ACQ (Asthma Control Questionnaire) y el ACT (Asthma Control Test $)^{5}$.

En cuanto a la reducción de los riesgos futuros, el riesgo de desarrollar exacerbaciones es el más importante debido su asociación con mayor

* Clínica Miguel de Servet, Integramédica.

** Centro de Salud Familiar Cardenal Raúl Silva Henríquez. San Pedro (ex Estación el Canelo). Puente Alto, Región Metropolitana. Chile. 
morbimortalidad, mayores costos de tratamiento ${ }^{6}$ y la posibilidad de llevar a una declinación progresiva en la función pulmonar ${ }^{7}$. Se han descrito una serie de factores de riesgo modificables de exacerbaciones, como la presencia de síntomas, el sobreuso de inhaladores de rescate, el uso inadecuado de corticoides inhalados (CDi), un $\mathrm{VEF}_{1}$ bajo el $60 \%$ del predicho, problemas psicosociales o socioeconómicos, tabaquismo, obesidad, eosinofilia, embarazo, etc ${ }^{2}$.

En la gran mayoría de los estudios clínicos controlados, los asmáticos son capaces de alcanzar un buen nivel de control del asma, lo que no se repite en los estudios llamados "de la vida real", en los que predominan los asmáticos con mal control clínico ${ }^{8}$. Es importante identificar a los pacientes con asma mal controlada ya que requieren revisión de su terapia e identificación de las causas de ello ${ }^{8}$. La falta de control clínico se asocia con peor calidad de vida ${ }^{9}$ y mayor utilización de recursos sanitarios ${ }^{3}$ y probablemente tiene causas multifactoriales que combinan componentes ambientales, fisiológicos y creencias o actitudes de los pacientes ${ }^{10-12}$. El grado de control del asma y la importancia relativa de los factores que contribuyen a ella varía localmen$t^{13}$, por lo que es primordial conocer la realidad local de cada centro de salud para así optimizar la terapia individual y también ayudar a desarrollar programas locales destinados a mejorar el grado de control en su conjunto. De ahí que el objetivo de este estudio fue evaluar el grado de control clínico del asma en un centro de atención primaria.

La comuna de Puente Alto tiene 6 centros de salud familiares (CESFAM), todos con salas ERA (enfermedades respiratorias agudas). Las salas ERA son una estrategia de salud diseñada centralmente para enfrentar los problemas diagnósticos y de manejo de algunas patologías respiratorias, incluyendo el asma ${ }^{14}$. Las salas ERA están constituidas por un equipo de profesionales de atención primaria que han sido capacitados por el Ministerio de Salud de Chile y que disponen de protocolos de manejo estandarizados ${ }^{15}$. La sala ERA del CESFAM Cardenal Raúl Silva Henríquez de la comuna de Puente Alto funciona desde el año 2004 y actualmente está compuesta por un médico general y dos kinesiólogas.

El objetivo principal de este estudio fue evaluar el grado de control clínico del asma de los pacientes que se manejan en la sala ERA del CESFAM Cardenal Raúl Silva Henríquez en cada visita programada. El objetivo secundario fue establecer la presencia de factores de riesgo modificables de exacerbaciones en la misma población.

\section{Material y Método}

Se revisaron las fichas electrónicas y cartolas clínicas de todas las visitas programadas a la sala ERA entre noviembre de 2014 y junio de 2015 de los pacientes asmáticos que cumplían los siguientes criterios de inclusión: edad de 20 años y más; en control en la sala ERA por asma desde hace más de 1 mes; con al menos un control médico en la sala ERA posterior al ingreso; sin exacerbación en el momento de la visita; con al menos un registro espirométrico.

Para los fines de este estudio se definió exacerbación en el momento de la visita si el paciente estaba usando algún antibiótico y/o corticoide debido al asma. Se excluyó del análisis las visitas con el paciente exacerbado ya que objetivo era la evaluación del grado de control de los pacientes en su etapa estable.

De los registros clínicos se obtuvieron los datos demográficos, clínicos, espirométricos y de las evaluaciones hechas en cada visita programada.

La evaluación realizada en cada visita consistió en, además del examen clínico, la evaluación del uso de los inhaladores prescritos en la visita previa (tanto la adherencia como la técnica de inhalación), las consultas médicas no programadas debido al asma (tanto a médico como al servicio de urgencias) y el puntaje del Test de Control del Asma (ACT).

El cuestionario ACT fue desarrollado el año $2004^{16}$ y está diseñado para ser autocompletado por los pacientes. Consta de 5 preguntas, cada una con cinco posibles respuestas. Las preguntas están dirigidas a medir la limitación en las actividades de la vida diaria, la disnea, los síntomas nocturnos, el uso de la terapia de rescate y la percepción de autocontrol por parte del paciente, todo durante las últimas 4 semanas. Un puntaje de 25 significa que el asma está totalmente controlada; entre 20 y 24, parcialmente controlada y 19 o menos, no controlada ${ }^{13}$. La diferencia clínica mínima significativa se ha establecido en 3 pun$\operatorname{tos}^{17}$. Nuestro análisis se enfocará principalmente en el asma no controlada.

Los factores de riesgo modificables de exacerbaciones analizados fueron el estado nutricional, el hábito tabáquico, el uso de corticoides inhalados y el porcentaje predicho del $\mathrm{VEF}_{1}$ post broncodilatador. Los tres primeros fueron registrados en cada visita y la función pulmonar de la espirometría más cercana a la visita.

En relación a los aspectos éticos, en este estudio se realizó una revisión de datos de las fichas y cartolas clínicas resguardando absolutamente 
la confidencialidad de los datos personales de los participantes. La metodología para que esto ocurriese fue la siguiente: de los cuatro autores del estudio, tres de ellos (LF, JH y LM) realizaron los controles en la sala ERA. Al final del período preestablecido, uno de ellos (LF) recolectó los datos, transformando cada visita en un número correlativo, con el que se encriptó la identificación de los pacientes con los datos recolectados. Posteriormente el cuarto autor (GA), externo al CESFAM, revisó y analizó los datos teniendo a la vista los números correlativos de cada visita.

Para el análisis estadístico se utilizó la prueba de $\chi^{2}$ para establecer la significancia estadística de las diferencias entre las variables categóricas considerando diferencia significativa con valores de $\mathrm{p}<0,05$.

\section{Resultados}

De las 281 visitas programadas de pacientes asmáticos entre noviembre de 2014 y junio de 2015, 19 no tenían control médico posterior al ingreso a la sala ERA, 5 fueron hechas con menos de un mes de la anterior, 10 no tenían registro de espirometría, en 2 pacientes no se pudo realizar el ACT y en una visita el paciente no autocompletó el ACT. 8 visitas correspondieron a una exacerbación leve.

Se incluyeron en el análisis 236 visitas, que correspondieron a 183 pacientes. En la Tabla 1 se muestran las características de los pacientes en las visitas. El 79,2\% fueron mujeres, de edad promedio 53,3 años (mínimo 23 y máximo 88 años). El tiempo promedio de seguimiento de los pacientes en la sala ERA fue de 6 años.

El 55,9\% había fumado alguna vez, con un índice paquete/año (IPA) promedio de 15,2. De ellos, el 31\% seguía fumando (IPA: 20,3), lo que equivale al $17,4 \%$ de la población total analizada. El 10,6\% tenía un índice de masa corporal (IMC) normal, $41,5 \%$ sobrepeso y $47,9 \%$ era obeso. El $69 \%$ de los pacientes tenían al menos una comorbilidad crónica. Un 48,7\% tenía hipertensión arterial, seguido de diabetes mellitus $(23,7 \%)$, dislipidemia $(16,1 \%)$ e insuficiencia renal crónica $(9,3 \%) .35,6 \%$ tenía una sola comorbilidad, $21,2 \%$ dos comorbilidades, $10,2 \%$ tres comorbilidades y $2,1 \%$ cuatro comorbilidades. El $60,6 \%$ tenía una espirometría obstructiva. De ellas, $54,5 \%$ era modificable con el broncodilatador y $8,4 \%$ tenía un $\mathrm{VEF}_{1}$ post broncodilatador menor al $60 \%$ del predicho $(5,1 \%$ de la población total). E1 98,3\% de los pacientes tenía prescrito un CDi, de los cuales el 93,1\% refería estar ocupando en cada visita. El 81\% de los pacientes que usaba la terapia tenía indicado monoterapia con CDi y el $19 \%$ terapia combinada de CDi más un agonista beta adrenérgico de acción prolongada. El 7,2 \% de los pacientes había acudido a consulta médica no programada por el asma durante las últimas 4 semanas y el 38,6\% había asistido al servicio de urgencia por asma durante el año previo $(63,7 \%$ de ellos lo había hecho más de una vez).

El ACT promedio de todas las visitas fue de

Tabla 1. Características de la población asmática estudiada en las visitas

\begin{tabular}{|c|c|c|c|c|c|}
\hline & & Control total & Control parcial & Sin control & Total \\
\hline Género & $\begin{array}{l}\text { Masculino (\%) } \\
\text { Femenino (\%) }\end{array}$ & $\begin{array}{l}12,5 \\
87,5\end{array}$ & $\begin{array}{l}23,7 \\
76,3\end{array}$ & $\begin{array}{l}20,5 \\
79,5\end{array}$ & $\begin{array}{l}20,7 \\
79,3\end{array}$ \\
\hline Edad & $\begin{array}{l}\text { Promedio (años) } \\
\text { DS*** }\end{array}$ & $\begin{array}{l}54,2 \\
12,1\end{array}$ & $\begin{array}{l}53,2 \\
12,9\end{array}$ & $\begin{array}{l}53,2 \\
12,3\end{array}$ & $\begin{array}{l}53,3 \\
12,5\end{array}$ \\
\hline $\mathrm{IMC}^{*}$ & $\begin{array}{l}\text { Normal (\%) } \\
\text { SP }^{\dagger} / \text { Obeso (\%) }\end{array}$ & $\begin{array}{l}12,5 \\
87,5\end{array}$ & $\begin{array}{l}13,7 \\
86,3\end{array}$ & $\begin{array}{r}8,3 \\
91,7\end{array}$ & $\begin{array}{l}10,6 \\
89,4\end{array}$ \\
\hline Tabaco & $\begin{array}{l}\text { Sí (\%) } \\
\text { No (\%) }\end{array}$ & $\begin{array}{l}66,7 \\
33,3\end{array}$ & $\begin{array}{l}53,7 \\
46,3\end{array}$ & $\begin{array}{l}55,3 \\
44,7\end{array}$ & $\begin{array}{l}55,9 \\
44,1\end{array}$ \\
\hline Comorbilidades & $\begin{array}{l}\text { Sí (\%) } \\
\text { No (\%) }\end{array}$ & $\begin{array}{l}70,8 \\
29,1\end{array}$ & $\begin{array}{l}68,8 \\
31,2\end{array}$ & $\begin{array}{l}68,9 \\
31,1\end{array}$ & $\begin{array}{l}69,1 \\
30,9\end{array}$ \\
\hline $\mathrm{VEF}_{1} * *$ & $\begin{array}{l}\geq 80(\%) \\
<80(\%)\end{array}$ & $\begin{array}{r}100 \\
0\end{array}$ & $\begin{array}{l}82,5 \\
17,5\end{array}$ & $\begin{array}{l}83,3 \\
16,7\end{array}$ & $\begin{array}{l}84,7 \\
15,3\end{array}$ \\
\hline Tratamiento & $\begin{array}{l}\text { Con } \mathrm{CDi}^{\dagger \dagger}(\%) \\
\text { Sin CDi }(\%)\end{array}$ & $\begin{array}{l}79,2 \\
20,8\end{array}$ & $\begin{array}{r}91,2 \\
8,3\end{array}$ & $\begin{array}{r}93,9 \\
6,1\end{array}$ & $\begin{array}{r}91,5 \\
8,5\end{array}$ \\
\hline
\end{tabular}

*IMC: Índice de masa corporal; **Porcentaje del predicho; ***DS: desviación estándar; ${ }^{\dagger}$ SP: Sobrepeso; ${ }^{\dagger \dagger C D i: ~ C o r-~}$ ticoides inhalados. 


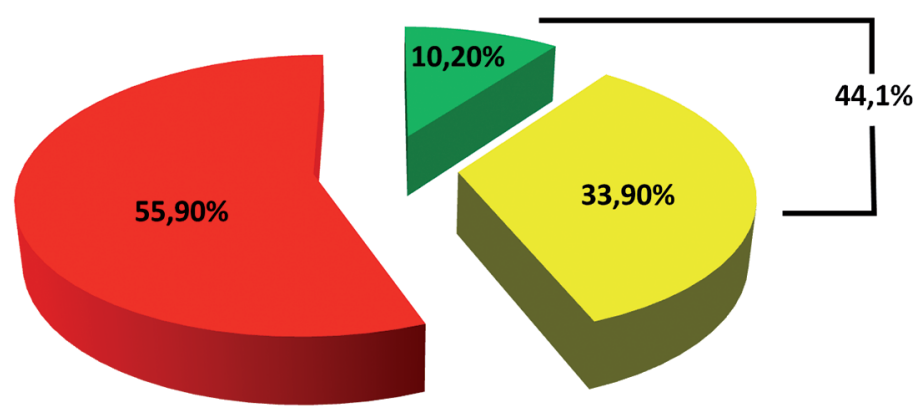

- Totalmente controlada
Parcialmente controlada
No controlada
Figura 1. Distribución en porcentaje del grado de control del asma en el total de visitas.
18,3. El 10,2\% de los asmáticos tenía un puntaje de ACT de 25 puntos (totalmente controlada), el $33,9 \%$ entre 20 y 24 (parcialmente controlada) y el $55,9 \%$ menor a 20 puntos (mal controlada) (Figura 1).

La Tabla 2 muestra las asociaciones entre el grado de control del asma y algunas características de los pacientes en las visitas. No hubo asociación entre el mal control del asma con el sexo $(p=0,9)$, edad $(p=0,9)$, estado nutricional $(\mathrm{p}=0,3)$, hábito tabáquico $(\mathrm{p}=0,7)$, número de comorbilidades crónicas $(\mathrm{p}=0,73)$, severidad de la obstrucción bronquial $(\mathrm{p}=0,07)$, reversibilidad de la obstrucción bronquial $(\mathrm{p}=0,5)$, uso de corticoides inhalados $(p=0,1)$ ni con la frecuencia de controles médicos en la sala ERA $(p=0,1)$. Hubo asociación entre el deficiente control del asma y el mayor uso de recursos sanitarios (número de consultas en servicios de urgencias en el último año y/o consultas médicas por asma no programadas $)(p=0,009)$.

El $92 \%$ tenía al menos un factor de riesgo modificable de exacerbación. La obesidad fue el factor de riesgo más frecuente $(47,9 \%)$, seguido de los fumadores activos $(17,4 \%)$, los que no usaban corticoides inhalados $(6,9 \%)$ y los que tenían un porcentaje del $\mathrm{VEF}_{1}$ predicho menor al $60 \%(5,1 \%)$. El 15,7\% tenía dos factores de riesgo, siendo la obesidad y el tabaquismo activo la combinación más frecuente $(7,2 \%)$ seguida de la obesidad más el porcentaje del $\mathrm{VEF}_{1} \%$ predicho menor al $60 \%(3,4 \%)$.

\section{Discusión}

Los principales hallazgos de este estudio de la vida real realizado en pacientes con diagnóstico de asma en atención primaria fueron dos: primero, sobre el $50 \%$ de las visitas programadas
Tabla 2. Asociación entre el grado de control del asma y algunas características de los pacientes en cada visita

\begin{tabular}{|lc|}
\hline Característica & Valor de p \\
\hline Género & 0,94 \\
\hline Edad & 0,9 \\
\hline Estado nutricional & 0,35 \\
\hline Tabaquismo activo & 0,78 \\
\hline Severidad espirométrica & 0,07 \\
\hline Reversibilidad espirométrica & 0,54 \\
\hline Uso de corticoides inhalados & 0,14 \\
\hline Número de comorbilidades & 0,73 \\
\hline Uso de recursos sanitarios & 0,009 \\
\hline
\end{tabular}

encontró a los pacientes en condición de asma mal controlada. Segundo, en la gran mayoría de las visitas, los pacientes presentaban al menos un factor de riesgo modificable de exacerbación del asma.

Para la evaluación del control sintomático elegimos el cuestionario ACT por varias razones. A diferencia del cuestionario de GINA, tanto el ACT como el ACQ están ampliamente validados ${ }^{18}$; el ACT es más simple de utilizar ya que, a diferencia del ACQ, no requiere la evaluación de la función pulmonar ${ }^{19}$; el ACT es preferible al ACQ en la práctica clínica ya que el ACQ requiere aún de mayor validación ${ }^{19}$; el ACT responde a los cambios en el control del asma en el tiempo ${ }^{20}$.

Nuestro estudio mostró que en el $55,9 \%$ de las visitas, los pacientes tenían su asma no controlada y en sólo el 10,2\% el asma estaba totalmente controlada. Esta preponderancia de asmáticos no controlados está en concordancia con la gran mayoría de los estudios de la vida real que han 
investigado el grado de control del asma. Por ejemplo, Chapman et al., analizaron el grado de control del asma mediante un cuestionario desarrollado por las guías canadienses para el manejo del asma en 10.428 pacientes, encontrando que en el $59 \%$ de ellos estaba no controlada ${ }^{21}$. Un estudio de una muestra nacional en Canadá, que utilizó este mismo cuestionario, mostró un 19\% de asmáticos no controlados ${ }^{22}$. El estudio ARIAP (Asthma Insights and Reality in Asia-Pacific StudyAsia), que usó para evaluar el grado de control un índice de severidad de síntomas basado en las guías GINA 2003, encontró que el 35,3\% tenía el asma no controlada ${ }^{23}$. Si bien los resultados de estos estudios muestran, al igual que el nuestro, un predominio de asmáticos no controlados sobre los completamente controlados, el hecho de utilizar distintos instrumentos para evaluar el grado de control puede no tener los mismos significados. De hecho, se ha demostrado que el ACT, comparado con el instrumento de GINA, clasifica significativamente más pacientes como no controlados $^{8}$, lo que sugiere que la elección del instrumento podría tener diferentes efectos sobre el grado de control, tanto en el aspecto individual como en el poblacional. Otro aspecto importante a recalcar, ya que también influye al momento de hacer comparaciones, es que nuestro estudio, a diferencia de la mayoría de los estudios publicados, evaluó el grado de control del asma de cada visita programada y no de cada paciente.

De los estudios que han utilizado el ACT, Mintz y cols., evaluaron el control del asma en atención primaria encontrando un $58 \%$ de asmáticos no controlados ${ }^{24}$. Otro estudio en atención primaria $^{25}$ mostró que el $39,8 \%$ de los pacientes tenía el asma no controlada, aunque este estudio utilizó un valor de corte del ACT $<16$. El estudio REACT (Real-world Evaluation of Asthma Control and Treatment), que evaluó una muestra representativa nacional de EEUU reclutada vía web, encontró un $55 \%$ de asma no controlada ${ }^{26}$.

No existen muchos datos en nuestro país sobre el grado de control del asma. Un estudio de Vidal y cols., realizado en 220 adolescentes que se atendían en el nivel terciario en Santiago y que ocupó el ACT, mostró que el 46,3\% tenía su asma no controlada ${ }^{27}$. Este estudio, que arrojó resultados similares al nuestro, se realizó con el manejo hecho por especialistas, a diferencia del nuestro que se hizo con el manejo de la patología por profesionales de atención primaria. Esta concordancia en el grado de control entre los diferentes niveles de atención se ha observado también en países más desarrollados. Por ejemplo, un estudio realizado en pacientes atendidos en el nivel terciario encontró que el 64\% tenían el asma no controlada ${ }^{28}$ y el estudio CHARIOT, realizado en 1.009 asmáticos que se atendieron por especialistas en clínicas pulmonares en EEUU, mostró que el $81,9 \%$ de los pacientes tenían su asma no controlada ${ }^{29}$.

Datos del estudio AIRLA (Asthma Insights and Reality in Latin America), realizado en 11 países de América Latina, que incluyó a Chile, si bien no reportó los datos específicos de nuestro país, mostró que el 32,5\% tenía el asma no controla$\mathrm{da}^{30}$. Este estudio evaluó el grado de control del asma según un índice de severidad de síntomas basado en las guías GINA 2003 e incluyó niños y adultos. Si bien estos resultados difieren algo de los nuestros, hay que destacar la diferencia en el instrumento utilizado para establecer el grado de control del asma, en la inclusión de niños y que estos pacientes fueron reclutados mediante encuestas a los hogares, por lo que el perfil de los pacientes es diferente. Por último, un estudio llevado a cabo en países de América Latina, que no incluyó a Chile, encontró que el $8 \%$ de los asmáticos tenía su asma controlada ${ }^{31}$. Este estudio no comunicó los resultados de los asmáticos no controlados y utilizó el instrumento recomendado por las guías GINA para establecer el grado de control del asma. Si bien el porcentaje de asmáticos controlados es similar al nuestro, las diferencias metodológicas entre ambos no los hace del todo comparables.

Los hallazgos aportados por nuestro estudio a la evidencia disponible expuesta sugieren que, sin importar el instrumento utilizado para establecer el grado de control del asma en la vida real, un porcentaje importante de pacientes mantiene su asma no controlada.

El grado de control del asma se correlaciona fuertemente con índices de calidad de vida ${ }^{9}$ y el mantener un mal control se asocia con el desarrollo de exacerbaciones ${ }^{2}$, da cuenta de costos desproporcionados y representa una pesada carga socioeconómica ${ }^{3}$. En nuestro estudio, el mal control del asma se asoció significativamente con mayor uso de recursos sanitarios, dado por la suma de mayor número de consultas en servicios de urgencias y de consultas médicas no programadas por asma.

Diversos estudios han investigado sobre los posibles factores que influyen en el control del asma. Es así como se ha asociado al asma no controlada con el sexo femenino ${ }^{27}$, el bajo nivel educacional $^{32}$, la falta de acceso a los medicamentos y el no uso de $\mathrm{CDi}^{33}$, el uso no apropiado de los inhaladores ${ }^{34}$, tratamiento insuficiente ${ }^{4}$, sobrestimación del grado de $\operatorname{control}^{4}$, la falta de 
información sobre su enfermedad ${ }^{35}$, tabaquismo activo $^{21}$ y pasivo $^{36}$, el status socioeconómico ${ }^{37}$, la obesidad $^{38}$, entre otros.

Nuestro estudio no mostró asociación significativa entre el mal control del asma con el sexo, hábito tabáquico ni uso de corticoides inhalados. Probablemente, debido a que en nuestra muestra se observó predominio de mujeres sobre hombres (4:1), el sexo no resultó estar asociado al mal control del asma. En cuanto al tabaquismo activo, no todos los estudios han mostrado su asociación con el mal control del asma ${ }^{26}$, no obstante la similar prevalencia con la población general de tabaquismo activo en los asmáticos (alrededor del $20 \%)^{21,26,39}$. No queda clara la causa de esta falta de asociación vista en nuestro estudio ya que la cantidad de cigarrillos fumados por nuestros pacientes fue alto (20 paquetes/año en promedio) y es conocida la resistencia a los corticoides inducida por el tabaquismo ${ }^{40}$. En cuanto a la falta de asociación con el no uso de corticoides inhalados que arrojó nuestro estudio, probablemente se explique por la escasa cantidad de sujetos que no estaban usando dicha terapia $(7 \%)$.

Nuestro estudio tampoco encontró asociación entre la obesidad y la falta de control del asma. $\mathrm{Si}$ bien existen estudios que muestran dicha asociación ${ }^{38} \mathrm{y}$ otros que no ${ }^{13}$, la explicación más probable de esta discordancia en los resultados sea que la obesidad central, y no el exceso de grasa corporal, es la que se asocia con peor control del asma. Es conocida la limitación del IMC en cuanto a distinguir entre la masa grasa y la masa muscular y su bajo correlato con el depósito de grasa abdominal, que es lo que se asocia con disminución en la función pulmonar, síndrome metabólico, complicaciones cardiovasculares y posiblemente con peor control del asma ${ }^{18}$.

Los hallazgos de nuestro estudio comparado con los de la literatura, tan disímiles unos y tan concordantes otros, apoyan la observación de algunos autores en cuanto a que los factores que contribuyen a la falta de control del asma varían localmente ${ }^{13}$. Por esto mismo, nuestro estudio también exploró otras asociaciones, como el número de comorbilidades crónicas, severidad de la obstrucción bronquial, reversibilidad espirométrica de la obstrucción bronquial y la frecuencia de controles médicos en la sala ERA, no encontrando asociación significativa con ninguno de ellos. Estudios epidemiológicos han mostrado que el asma se asocia débilmente con las enfermedades cardiovasculares, diabetes mellitus, dislipidemia, depresión, osteoporosis y rinosinusitis $^{41}$ y se asocia fuertemente con el reflujo gastroesofágico (RGE), la sinusitis crónica y la rinitis alérgica ${ }^{42}$. En nuestro estudio, la presencia de RGE y sinusitis crónica está escasamente registrada en las fichas y cartolas clínicas, por lo que no se puede analizar con certeza asociación alguna. En cuanto a la hipertensión arterial (HTA), cerca de la mitad de nuestros pacientes tenía esta comorbilidad, pero no hubo asociación con el mal control del asma. Otros estudios han encontrado sólo una significación límite entre el asma y la $\mathrm{HTA}^{26}$. En cuanto a la asociación entre el número de comorbilidades y el grado de control del asma, los escasos estudios que lo han comunicado no han encontrado asociación ${ }^{43}$, al igual que el nuestro.

Los datos espirométricos en nuestro estudio no son, en su gran mayoría, concurrentes temporalmente a las visitas, por lo que no se puede establecer asociación cierta. Por último, la falta de asociación entre la frecuencia de controles médicos en la sala ERA y el grado de control también se ha detectado en otros estudios ${ }^{21}$.

Probablemente una de las principales razones que explican la falta de asociación de nuestro estudio es la homogeneidad de la muestra, lo que puede apreciarse en la Tabla 1. La gran mayoría de los pacientes fueron mujeres, de la misma edad, con sobrepeso u obesas, fumadoras y con comorbilidades.

En cuanto a la presencia de factores de riesgo modificables en las visitas no programadas, nuestros resultados arrojaron que cerca del $40 \%$ de los pacientes había acudido al servicio de urgencias durante el año previo y/o había asistido a consulta médica no programada por asma, probablemente debido a una exacerbación. Este porcentaje es inferior al de otros reportes ${ }^{22,23,30}$ debido probablemente a que mayoritariamente nuestros pacientes tenían un fenotipo de asma más bien leve, esto debido al bajo porcentaje que usaba terapia combinada (19\%).

Un hallazgo importante de nuestro estudio es el alto porcentaje de asmáticos no controlados a pesar del uso amplio de CDi. Este resultado contraintuitivo podría explicarse por el uso inadecuado del inhalador, la mala calidad de la droga utilizada o el dato no fidedigno entregado por los pacientes. Esto último no se puede descartar a priori ya que la mayoría de los estudios tanto nacionales ${ }^{44}$ como extranjeros $^{45}$, muestran la baja adherencia al tratamiento. Otra explicación podría ser la existencia de sesgo por indicación, manifestado por el hecho que pacientes con mal control del asma en la visita inmediatamente anterior, se les podría haber prescrito o corregido el uso de corticoides, no logrando aún el control sintomático en la visita incluida en el presente 
estudio. En todo caso, la metodología del estudio no permite una aproximación causal a este hallazgo.

Una alta proporción de nuestros pacientes $(92 \%)$ presentaba, en cada visita programada, al menos un factor de riesgo modificable de exacerbación, estando la obesidad presente en casi la mitad de las visitas, siendo esta de mayor proporción que en la mayoría de los estudios publicados ${ }^{18,38}$. El tabaquismo activo en nuestra población, como ya lo mencionamos, fue similar a la de los estudios publicados. Cerca del 16\% tenía dos factores de riesgo, siendo la obesidad y el tabaquismo activo la combinación más frecuente.

Este estudio tiene varias limitaciones. Primero, el diseño retrospectivo con todos sus sesgos y factores confundentes. Segundo, las limitaciones propias que se le han descrito al ACT en cuanto a sobreestimar el asma no controlada ${ }^{21}$. Tercero, los distintos criterios utilizados para establecer el diagnóstico de asma. Si bien los criterios internacionales no han cambiado mucho en los últimos años, en nuestro estudio no pudimos comprobar objetivamente el cumplimiento de los criterios usados en los pacientes para catalogarlos de asmáticos, por lo que podrían haberse incluido en el estudio pacientes con otras patologías como EPOC, bronquiectasias, etc. Cuarto, la falta de comprobación de los datos entregados por los pacientes, especialmente en lo referente a la adherencia al tratamiento, lo que podría influir nuestros resultados. Quinto, la falta de registro de comorbilidades que se sabe altera el grado de control, como el RGE y la sinusitis crónica, podría también haber influido en los resultados. Sexto, si bien el criterio de inclusión de pacientes con antigüedad de al menos un mes podría determinar un sesgo de inclusión, que podría potencialmente influir en los resultados. Fue necesario utilizar este criterio ya que el ACT considera los síntomas durante las cuatro semanas, tiempo mínimo para evaluar el grado de control.

En suma, los pacientes con diagnóstico de asma atendidos en este centro de atención primaria mantienen su asma mal controlada en la mayoría de las visitas, lo que se ha traducido en mayor uso de recursos sanitarios. Una gran cantidad de ellos presentan, además, alto porcentaje de factores de riesgo modificables de exacerbaciones, específicamente obesidad y tabaquismo activo. Se hace necesario, entonces, realizar una investigación más profunda con el fin de buscar las causas de este mal control y adecuar el manejo de estos pacientes poniendo énfasis en programas para bajar de peso y cese tabáquico.

\section{Bibliografía}

1.- PAPAIOANNOU A I, KOSTIKAS K, ZERVAS E, KOLILEKAS L, PAPIRIS S, GAGA M. Control of Asthma in Real Life: Still a Valuable Goal? Eur Respir Rev 2015; 24: 361-9.

2.- GLOBAL INITIATIVE FOR ASTHMA. Global Strategy for Asthma Management and Prevention. Update 2015. www.ginasthma.org

3.- THOMAS M, KAY S, PIKE J, WILLIAMS A, CARRANZA J R, HILLYER E V, et al. The Asthma Control Test (ACT) as a Predictor of GINA Guideline-Defined Asthma Control: Analysis of a Multinational CrossSectional Survey. Prim Care Respir J 2009; 18: 41-9.

4.- BOULET L P, PHILLIPS R, O'BYRNE P, BECKER A. Evaluation of Asthma Control by Physicians and patients: Comparison with Current Guidelines. Can Respir J 2002; 9: 417-23.

5.- CLOUTIER M M, SCHATZ M, CASTRO M, CLARK N, KELLY H W, MANGIONE-SMITH R, et al Asthma Outcomes: Composite Scores of Asthma Control. J Allergy Clin Immunol 2012; 129: S24-33.

6.- CUSTOVIC A, JOHNSTON S L, PAVORD I, GAGA M, FABBRI L, BEL E H, et al. EAACI Position Statement on Asthma Exacerbations and Severe Asthma. Allergy 2013; 68: 1520-31.

7.- O'BYRNE P M, PEDERSEN S, LAMM C J, TAN W C, BUSSE WW, on behalf of the START Investigators Group. Severe Exacerbations and Decline in Lung Function in Asthma. Am J Respir Crit Care Med 2009; 179: 19-24.

8.- KORN S, BOTH J, JUNG M, HÜBNER M, TAUBE C, BUHL R. Prospective Evaluation of Current Asthma Control Using ACQ and ACT Compared with GINA Criteria. Ann Allergy Asthma Immunol 2011; 107: 474-9.

9.- VOLLMER W M, MARKSON L E, O'CONNOR E, SANOCKI LL, FITTERMAN L, BERGER M, et al Association of Asthma Control with Health Care Utilization and Quality of Life. Am J Respir Crit Care Med 1999; 160: 1647-52.

10.- CHIPPS B E, SPAHN J D. What Are the Determinates of Asthma Control? J Asthma 2006; 43: 567-72.

11.- DE VRIES M P, VAN DEN BEMT L, LINCE S, MURIS J W M, THOONEN B P A, VAN SCHAYCK C P. Factors Associated with Asthma Control. J Asthma 2005; 42: 659-65.

12.- HERJAVECZ I, NAGY G B, GYURKOVITS K, MAGYAR P, DOBOS K, NAGY L, et al. Cost, Morbidity, and Control of Asthma in Hungary: The Hunair Study. J Asthma 2003; 40: 673-81.

13.- PRZYBYSZOWSKI M, BOCHENEK G. The Role of Questionnaires in the Assesment of Asthma Control. Pneumonol Alergol Pol 2015; 83: 220-8.

14.- GOBIERNO DE CHILE. Ministerio de Salud. Programa de Control de las Enfermedades Respiratorias del Adulto en Chile. 
15.- GOBIERNO DE CHILE. Ministerio de Salud. Guía Clínica Asma Bronquial del Adulto. Santiago: MINSAL, 2013.

16.- NATHAN R A, SORKNESS C A, KOSINSKI M, SCHATZ M, LI JT, MARCUS P, et al. Development of the Asthma Control Test: a Survey for Assesing Asthma Control. J Allergy Clin Immunol 2004; 113: 59-65.

17.- SCHATZ M, KOSINSKI M, YARLAS A S, HANLON J, WATSON ME, JHINGRAN P. The Minimally Important Difference of the Asthma Control Test. J Allergy Clin Immunol 2009; 124: 719-23.

18.- CAPELO A V, DE FONSECA V M, MARQUES PEIXOTO M V, DE CARVALHO S R, GARCÍA GUERINO L. Central Obesity and Other Factors Associated with Uncontrolled Asthma in Women. Allergy Asthma Clin Immunol 2015; 11: 12.

19.- JIA C E, ZHANG H P, LV Y, LIANG R, JIANG Y Q, POWELL H, et al. The Asthma Control Test and Asthma Control Questionnaire for Assessing Asthma Control: Systematic Review and Meta-analysis. J Allergy Clin Immunol 2013; 131: 695-703.

20.- SCHATZ M, SORKNESS C A, LI J T, MARCUS P, MURRAY J J, NATHAN R A, et al. Asthma Control Test: Reliability, Validity, and Responsiveness in Patients not Previously Followed by Asthma Specialists. J Allergy Clin Immunol 2006; 117: 549-56.

21.- CHAPMAN K R, BOULET L P, REA R M, FRANSSEN E. Suboptimal Asthma Control: Prevalence, Detection and Consequences in General Practice. Eur Respir J 2008; 31: 320-5.

22.- CHAPMAN K R, ERNST P, GRENVILLE A, DEWLAND P, ZIMMERMAN S. Control of Asthma in Canada: Failure to Achieve Guideline Targets. Can Respir J 2001;8 (Suppl A): 35A-40A.

23.- LAI C K W, DE GUIA T S, KIM Y Y, KUO S H, MUKHOPADHYAY A, SORIANO J B, et al; on behalf of the Asthma Insights and Reality in Asia-Pacific Steering Committee. Asthma control in the Asia-Pacific region: The Asthma Insights and Reality in Asia-Pacific Study. J Allergy Clin Immunol 2003; 111: 263-8.

24.- MINTZ M, GILSENAN A W, BUI C L, ZIEMIECKI R, STANFORD R H, LINCOURT W, et al. Assessment of asthma control in primary care. Curr Med Res Opin 2009; 25: 2523-31.

25.- AL-ZAHRANI J M, AHMAD A, AL-HARBI A, KHAN A M, AL-BADER B, BAHAROON S, et al. Factors associated with poor asthma control in the outpatient clinic setting. Ann Thorac Med 2015; 10: 100-4.

26.- PETERS S P, JONES C A, HASELKORN T, MINK D R, VALACER D J, WEISS S T. Real-world Evaluation of Asthma Control and Treatment (REACT): Findings from a national Web-based survey. J Allergy Clin Immunol 2007; 119: 1454-61.

27.- VIDAL A, UBILLA C, DUFFAU G. Control de Asma en Adolescentes. Rev Med Chile 2008; 136: 859-66.

28.- AL-JAHDALI H H, AL-HAJJAJ M S, ALANEZI M
O, ZEITONI M O, AL-TASAN T H. Asthma control assessment using asthma control test among patients attending 5 tertiary care hospitals in Saudi Arabia. Saudi Med J 2008; 29: 714-7.

29.- MARCUS P, ARNOLD R J G, EKINS S, SACCO P, MASSANARI M, YOUNG S S, et al; on behalf of the CHARIOT Study Investigators. A retrospective randomized study of asthma control in the US: results of the CHARIOT study. Curr Med Res Opin 2008; 24: 3443-52.

30.- NEFFEN H, FRITSCHER C, CUEVAS SCHACHT F, LEVY G, CHIARELLA P, SORIANO J B, et al. Asthma control in Latin America: the Asthma Insights and Reality in Latin America (AIRLA) survey. Rev Panam Salud Publica 2005; 17: 191-7.

31.- NATHAN R A, THOMPSON P J, PRICE D, FABBRI L M, SALVI S, GONZÁLEZ-DÍAZ S, et al. Taking Aim at Asthma Around the World: Global Results of the Asthma Insight and Management Survey in the AsiaPacific Region, Latin America, Europe, Canada and the United States. J Allergy Clin Immunol Pract 2015; 3: 734-42.

32.- STANFORD R H, GILSENAN A W, SIEMIECKI R, ZHOU X, LINCOURT W R, ORTEGA H. Predictors of Uncontrolled Asthma in Adult and Pediatric Patients: Analysis of the Asthma Control Characteristics and Prevalence Survey Studies (ACCESS). J Asthma 2010; 47: 257-62.

33.- DALCIN P T R, MENEGOTTO D M, ZANONATO A, FRANCISCATTO L, SOLIMAN F, FIGUEIREDO $\mathrm{M}$, et al Factors associated with uncontrolled asthma in Porto Alegre, Brasil. Braz J Med Biol Res 2009; 42: 1097-103.

34.- AL-JAHDALI H, ANWAR A, AL-HARBI A, BAHAROON S, HALWANI R, AL SHIMEMERI A, et al. Factors associated with patient visits to the emergency department for asthma therapy. BMC Pulm Med 2012; 12: 80 .

35.- AL-JAHDALI H H, AL-ZAHRANI A I, AL-OTAIBI S T, HASSAN I S, AL-MOAMARY M S, AL-DUHAIM A $S$, et al. Perception of the role of inhaled corticosteroids and factors affecting compliance among asthmatic adult patients. Saudi Med J 2007; 28: 569-73.

36.- JINDAL S K, GUPTA D, SINGH A. Indices of Morbidity and Control of Asthma in Adult Patients Exposed to Environmental Tobacco Smoke. Chest 1994; 106: 746-9.

37.- ZAIHRA T, ERNST P, TAMBLYN R, AHMED S Tailoring interventions: identifying predictors of poor asthma control. Ann Allergy Asthma Immunol 2015; 114: 485-91.

38.- MOSEN D M, SCHATZ M, MAGID D J, CAMARGO $\mathrm{C}$ A. The relationship between obesity and asthma severity and control in adults. J Allergy Clin Immunol 2008; 122: 507-11.

39.- RABE K F, ADACHI M, LAI C K W, SORIANO J B, 
VERMEIRE P A, WEISS K B, et al. Worldwide severity and control of asthma in children and adults: The global Asthma Insights and Reality surveys. J Allergy Clin Immunol 2004; 114: 40-7.

40.- TOMLINSON J E, MCMAHON A D, CHAUDHURI R, THOMPSON J M, WOOD S F, THOMSON N C. Efficacy of low and high dose inhaled corticosteroid in smokers versus non-smokers with mild asthma. Thorax 2005; 60: 282-7.

41.- CAZZOLA M, CALZETTA L, BETTONCELLI G, NOVELLI L, CRICELLI C, ROGLIANI P. Asthma and comorbid medical illness. Eur Respir J 2011; 38: 42-9.

42.- CAZZOLA M, SEGRETI A, CALZETTA L, ROGLIA-
NI P. Comorbidities of asthma: current knowledge and future research needs. Curr Opin Pulm Med 2013; 19 : 36-41.

43.- WARDZYNSKAA, KUBSIK B, KOWALSKI M L. Comorbidities in elderly patients with asthma: Association with control of the disease and concomitant treatment. Geriatr Gerontol Int 2015; 15: 902-9.

44.- RIQUELME M, RIQUELME R, MARTÍNEZ D. Experiencia de un Programa de Control de Asma Bronquial en Puerto Montt, Chile. Rev Chil Enferm Respir 2006; 22: 93-7.

45.- SUMINO K, CABAN M D. Medication adherence in asthma patients. Curr Opin Pulm Med 2013; 19: 49-53.

Correspondencia a:

Dr. Gonzalo Alvear T.

Clínica Miguel de Servet, Integramédica.

Almirante Pastene 190, Providencia. Santiago de Chile.

E mail: galveart@hotmail.com 